\title{
Some applications of metacyclic 2-groups of negative type
}

\author{
Sanaa Mohamed Saleh Omer ${ }^{\mathrm{a}}$, Nor Haniza Sarmin ${ }^{\mathrm{b}, *}$, Ahmad Erfanian ${ }^{\mathrm{c}}$ \\ a Department of Mathematics, Faculty of Science, University of Benghazi, Benghazi, Libya \\ b Department of Mathematical Sciences, Faculty of Science, Universiti Teknologi Malaysia, Johor Bahru, \\ Malaysia \\ c Department of Mathematics and Centre of Excellence in Analysis on Algebraic Structures, \\ Ferdowsi University of Mashhad, Mashhad, Iran
}

*Corresponding author, e-mail: nhs@utm.my

Received 31 Aug 2014

Accepted 20 Jul 2016

\begin{abstract}
The probability that two random elements commute in a finite group $G$ is the quotient of the number of commuting elements and $|G|^{2}$. Consider a set $S$ consisting of all subsets of commuting elements of $G$ of size two that are in the form $(a, b)$ where $a$ and $b$ commute and $\operatorname{lcm}(|a|,|b|)=2$. The probability that a group element fixes $S$ is the number of orbits under the group action on $S$ divided by $|S|$. In this paper, the probability that a group element fixes a set $S$ under regular action is found for metacyclic 2-groups of negative type of nilpotency class two and of class at least three. The results obtained from the sizes of the orbits are then applied to the generalized conjugacy class graph.
\end{abstract}

KEYWORDS: commutativity degree, group action, conjugacy class graph

MSC2010: 20P05 20B40 97K30 58E40

\section{INTRODUCTION}

Throughout this paper, $\Gamma$ denotes a simple undirected graph and $G$ denotes a finite non-abelian group. The probability that a pair of elements $x$ and $y$ selected randomly from a group $G$ commute is called the commutativity degree ${ }^{1}$. A generalization of this is the probability that a group element fixes a $\operatorname{set}^{2}$.

Bertram et $\mathrm{al}^{3}$ introduced a new graph related to the conjugacy class. The vertices of this graph are non-central conjugacy classes, i.e., $V(\Gamma)=K(G)-$ $Z(G)$, where $K(G)$ is the number of conjugacy class of a group and $Z(G)$ is the centre of group $G$. A pair of vertices of this graph is connected by an edge if their cardinalities are not coprime. Omer et $\mathrm{al}^{4}$ extended the work on this conjugacy class graph by introducing a generalized conjugacy class graph whose vertices are non-central orbits under group action on a set.

Beuerle classified the metacyclic $p$-groups as being either metacyclic p-groups of nilpotency of class two or metacyclic $p$-groups of nilpotency of class at least three ${ }^{5}$. Based on Beuerle's classification, the metacyclic $p$-groups of nilpotency class two are partitioned into two families of non-isomorphic p-groups (types (i) and (ii) in the following list). The metacyclic 2-groups of negative type of class at least three are partitioned into eight families ${ }^{5}$. Types (iii)-(x) in the following list are some of the negative types that are considered here.

(i) $G \cong\left\langle a, b: a^{p^{\alpha}}=1, b^{p^{\beta}}=1,[a, b]=a^{p^{\alpha-\gamma}}\right\rangle$, where $\alpha, \beta, \gamma \in \mathbb{N}, \alpha \geqslant 2 \gamma, \beta \geqslant \gamma \geqslant 1$;

(ii) $G \cong\left\langle a, b: a^{4}=1, b^{2}=[b, a]=a^{-2}\right\rangle$, namely, a quaternion of order $8, Q_{8}$.

(iii) $G \cong\left\langle a, b: a^{2^{\alpha}}=1, b^{2}=a^{2^{\alpha-1}},[b, a]=a^{-2}\right\rangle$, where $\alpha \geqslant 3$;

(iv) $G \cong\left\langle a, b: a^{2^{\alpha}}=b^{2}=1,[b, a]=a^{-2}\right\rangle$, where $\alpha \geqslant 3$

(v) $G \cong\left\langle a, b: a^{2^{\alpha}}=b^{2}=1,[b, a]=a^{2^{\alpha-1}-2}\right\rangle$, where $\alpha \geqslant 3$;

(vi) $G \cong\left\langle a, b: a^{2^{\alpha}}=b^{2^{\beta}}=1,[b, a]=a^{-2}\right\rangle$, where $\alpha \geqslant 3, \beta>1$;

(vii) $G \cong\left\langle a, b: a^{2^{\alpha}}=b^{2^{\beta}}=1,[b, a]=a^{2^{\alpha-1}-2}\right\rangle$, where $\alpha \geqslant 3, \beta>1$;

(viii) $G \cong\left\langle a, b: a^{2^{\alpha}}=1, b^{2^{\beta}}=a^{2^{\alpha-1}},[b, a]=\right.$ $\left.a^{2^{\alpha-\gamma}-2}\right\rangle$, where $\alpha-\gamma>1, \beta>\gamma>1$;

(ix) $G \cong\left\langle a, b: a^{2^{\alpha}}=b^{2^{\beta}}=1,[b, a]=a^{2^{\alpha-\gamma}-2}\right\rangle$, where $\alpha-\gamma>1, \beta \geqslant \gamma>1$;

(x) $G \cong\left\langle a, b: a^{2^{\alpha}}=1, b^{2^{\beta}}=a^{2^{\alpha-1}},[b, a]=a^{-2}\right\rangle$, where $\alpha \geqslant 3, \beta>1$. 
This paper is divided into three sections. The first section focuses on some background topics in graph theory and algebra. The second section provides some earlier and recent publications that are related to the probability that a group element fixes a set and conjugacy class graph. In the third section, we present our main results which include the probability that an element of metacyclic 2group element fixes a set and generalized conjugacy class graph.

\section{PRELIMINARIES}

Theorem 1 (Ref. 2) Let $G$ be a finite group. Let $S$ be a set of elements of $G \times G$ of size two in the form $(a, b)$ where $a$ and $b$ commute and $\operatorname{lcm}(|a|,|b|)=2$. Let $\Omega$ be the set of all subsets of commuting elements of $G$ of size two and $G$ acts on $\Omega$. Then the probability that an element of a group fixes a set is given by

$$
P_{G}(\Omega)=\frac{K(\Omega)}{|\Omega|},
$$

where $K(\Omega)$ is the number of orbits of $\Omega$ in $G$.

The work in Ref. 2 has then been extended by finding the probability for some finite non-abelian groups including the dihedral, quaternion, symmetric, and alternating groups ${ }^{2,6,7}$. In this paper, the probability that a metacyclic 2-group element fixes a set is computed. The obtained results are then applied to generalized conjugacy class graph by using the sizes of the orbits that are obtained under group action on a set. Some related works on conjugacy class graph include Bianchi et $\mathrm{al}^{8}$ who studied the regularity of the graph related to conjugacy classes and provided some results. Moreto et $\mathrm{al}^{9}$ classified the groups in which conjugacy classes sizes are not coprime for any five distinct classes. You et $\mathrm{al}^{10}$ classified the groups in which conjugacy classes are not set-wise relatively prime for any four distinct classes. Moradipour et al ${ }^{11}$ used the graph related to conjugacy classes to find some graph properties of some finite metacyclic 2-groups. Recently, Omer et $\mathrm{al}^{4}$ introduced generalized conjugacy class graph, given in the following definition.

Definition 1 Let $G$ be a finite group and $\Omega$ a set of $G$. Let $A$ be the set of commuting elements in $\Omega$, i.e., $A=\{\omega \in \Omega: \omega g=g \omega, g \in G\}$. Then the generalized conjugacy class graph $\Gamma_{G}^{\Omega_{\mathrm{c}}}$ is defined as a graph whose vertices are non-central orbits under group action on a set, i.e., $\left|V\left(\Gamma_{G}^{\Omega_{\mathrm{c}}}\right)\right|=K(\Omega)-|A|$. Two vertices $\omega_{1}$ and $\omega_{2}$ in $\Gamma_{G}^{\Omega_{\mathrm{c}}}$ are adjacent if their cardinalities are not coprime, i.e., $\operatorname{gcd}\left(\left|\omega_{1}\right|,\left|\omega_{2}\right|\right) \neq$ 1.
Moreover, the generalized conjugacy class graph is determined for the symmetric groups and alternating groups ${ }^{6}$.

\section{MAIN RESULTS}

This section consists of two parts. The first part focuses on the probability that an element of metacyclic 2-group fixes a set, while the second part relates the obtained results to generalized conjugacy class graph.

Throughout this section, let $S$ be a set of elements of $G \times G$ of size two in the form $(a, b)$, where $a$ and $b$ commute and $\operatorname{lcm}(|a|,|b|)=2$. Let $\Omega$ be the set of all subsets of commuting elements of $G$ of size two.

\section{The probability that a metacyclic 2-group element fixes a set}

In this section, the probability that a group element fixes a set is found for metacyclic $p$-groups of negative type of nilpotency of class two, starting with type (i).

Theorem 2 Let $G$ be a group of type (i). If $G$ acts regularly on $\Omega$, then

$$
P_{G}(\Omega)= \begin{cases}\frac{5}{11}, & \beta=\gamma=1, \alpha<3, \\ \frac{1}{2}, & \text { otherwise. }\end{cases}
$$

Proof: The elements of $\Omega$ can be expressed as follows. There are four elements in the form $\left(1, a^{i} b\right), 0 \leqslant i \leqslant 2^{\alpha}$, four elements are in the form $\left(a^{2^{\alpha-1}}, a^{i} b\right), 0 \leqslant i \leqslant 2^{\alpha}$, two elements are in the form $\left(a^{i} b, a^{i+2^{\alpha-1}} b\right)$, and one element is in the form $\left(1, a^{2^{\alpha}}\right)$, from which it follows that $|\Omega|=$ 11. Thus by regular action there are four orbits of the form $\left\{\left(1, a^{i} b\right),\left(a^{2^{\alpha-1}}, a^{i} b\right)\right\}, 0 \leqslant i \leqslant 2^{\alpha}$ and one orbit of $\left\{\left(1, a^{2^{\alpha}}\right),\left(a^{i} b, a^{2^{\alpha-1}+i} b\right)\right\}, 0 \leqslant i \leqslant 2^{\alpha}$. Using Theorem $1, P_{G}(\Omega)=5 /|\Omega|$. In the case that $\alpha>3$, there are six elements in $\Omega$. Thus by regular action, there are two orbits in the form $\left\{\left(1, a^{2^{\alpha-1} i} b^{2^{\beta-1}}\right),\left(a^{2^{\alpha-1}}, a^{2^{\alpha-1} i} b^{2^{\beta-1}}\right)\right.$ and one orbit is in the form $\left(1, a^{2^{\alpha-1}}\right),\left(b^{2^{\beta-1}}, a^{2^{\alpha-1}} b^{2^{\beta-1}}\right)$. Using Theorem $1, P_{G}(\Omega)=3 /|\Omega|$.

Theorem 3 Let $G$ be a group of type (ii). If $G$ acts regularly on $\Omega$, then $P_{G}(\Omega)=1$.

Proof: There is only one element in $\Omega$, namely, $\left(1, a^{2}\right)$. Thus when $G$ acts regularly on $\Omega$ there is only one orbit which is $\Omega$ itself. Using Theorem 1 , $P_{G}(\Omega)=1$.

Next, the probability that a metacyclic 2-group element fixes a set of class of at least three is found, starting with type (iii). 
Theorem 4 Let $G$ be a group of type (iii). If $G$ acts regularly on $\Omega$, then $P_{G}(\Omega)=\frac{5}{11}$.

Proof: If $G$ acts regularly on $\Omega$, then there exists an element $g \in G$ such that $g \omega_{1}=\omega_{2}$ for $\omega_{1}, \omega_{2} \in$ $\Omega$. The elements of $\Omega$ are as follows: there is only one element in the form $\left(1, a^{2^{\alpha-1}}\right)$, four elements are in the form $\left(a^{2^{\alpha-1}}, a^{2^{\alpha-3} i} b\right)$, where $i$ is odd and $0 \leqslant i \leqslant 2^{\alpha}$, four elements are in the form $\left(1, a^{2^{\alpha-3} i} b\right)$ where $i$ is odd and $0 \leqslant i \leqslant 2^{\alpha}$, and two elements are in the form $\left(a^{2^{\alpha-3} i} b, a^{2^{\alpha-3} i+2^{\alpha-1}} b\right)$, $0 \leqslant i \leqslant 2^{\alpha}$ and $i$ is odd. From this it follows that $|\Omega|=11$. By regular action, there exists an element $g \in G$ for $\omega_{1}, \omega_{2} \in \Omega$ such that $g \omega_{1}=\omega_{2}$. Thus there are four orbits of the form $\left\{\left(1, a^{2^{\alpha-3} i} b\right),\left(a^{2^{\alpha-1}}, a^{\alpha^{\alpha-3} i+2^{\alpha-1}} b\right)\right\}, 0 \leqslant i \leqslant 2^{\alpha}$ where $i$ is odd, and one orbit of $\left\{\left(a^{2^{\alpha-3} i} b, a^{2^{\alpha-3} i+2^{\alpha-1}} b\right)\right\}, 0 \leqslant$ $i \leqslant 2^{\alpha}$ and $i$ is odd. Using Theorem $1, P_{G}(\Omega)=$ $5 /|\Omega|$, as claimed.

Remark 1 The probability that an element of a metacyclic 2-group of type (iv) is the same as the probability of the metacyclic 2-group of type (iii).

Next, the probability that a metacyclic 2-group element of type (v) fixes a set is determined.

Theorem 5 Let $G$ be a group of type (v). If $G$ acts regularly on $\Omega$, then

$$
P_{G}(\Omega)= \begin{cases}\frac{9}{21}, & \alpha=3 \\ \frac{1}{2}, & \text { otherwise }\end{cases}
$$

Proof: In the case that $\alpha=3$, the elements of $\Omega$ are as follows. One element is in the form $\left(1, a^{2^{\alpha-1}}\right)$, there are $2^{\alpha}$ elements in the form $\left(a^{2^{\alpha-1}}, a^{i} b\right), 0 \leqslant i \leqslant 2^{\alpha}$, there are $2^{\alpha}$ elements in the form $\left(1, a^{i} b\right), 0 \leqslant i \leqslant 2^{\alpha}$ and $2^{\alpha-1}$ elements in which $\omega \in \Omega$ is in the form $\left(a^{i} b, a^{i+2^{\alpha-1}} b\right), 0 \leqslant$ $i \leqslant 2^{\alpha}$. From this it follows that $|\Omega|=2^{\alpha+1}+$ $2^{\alpha-1}+1=21$. By regular action, $g \omega_{1}=\omega_{2}$ which means that $g\left(1, a^{i} b\right)=\omega_{2}$. Hence $g$ must be of order one or two. Hence there are eight orbits of $\left\{\left(1, a^{i} b\right),\left(a^{2^{\alpha-1}}, a^{i+2^{\alpha-1}} b\right)\right\}, 0 \leqslant i \leqslant 2^{\alpha}$ and one orbit is $\left\{\left(1, a^{2^{\alpha-1}}\right),\left(a^{i} b, a^{i+2^{\alpha-1}} b\right)\right\}, 0 \leqslant i \leqslant 2^{\alpha}$. Hence $P_{G}(\Omega)=3 /|\Omega|$. In the case that $\alpha>3$, there are two orbits of size two and in the form $\left\{\left(1, a^{2^{\alpha-1} i}\right),\left(a^{2^{\alpha-1}}, a^{2^{\alpha-1} i} b\right)\right\}, 0 \leqslant i \leqslant 2^{\alpha}$ and one orbit represented by $\left\{\left(1, a^{2^{\alpha-1}}\right),\left(b, a^{2^{\alpha-1}} b\right)\right.$. Using Theorem $1, P_{G}(\Omega)=3 /|\Omega|$.

Theorem 6 Let $G$ be a group of type (vi). If $G$ acts regularly on $\Omega$, then $P_{G}(\Omega)=\frac{1}{2}$.
Proof: The elements of $\Omega$ are described as follows. There are two elements in the form $\left(1, a^{2^{\alpha-1} i} b^{2^{\beta-1}}\right), 0 \leqslant i \leqslant 2^{\alpha}$, two elements are in the form $\left(a^{2^{\alpha-1}}, a^{2^{\alpha-1} i} b^{2^{\beta-1}}\right), 0 \leqslant i \leqslant 2^{\alpha}$, one element is in the form $\left(b^{2^{\beta-1}}, a^{2^{\alpha-1}} b^{2^{\beta-1}}\right)$, and one element is in the form $\left(1, a^{a^{\alpha-1}}\right)$. Thus $|\Omega|=6$. When $G$ acts regularly on $\Omega$ there exists $g \in G$ and $\omega_{1}, \omega_{2} \in \Omega$ such that $g \omega_{1}=\omega_{2}$. To obtain an element of $\Omega$, $g$ here must be of order one or two. Thus there are two orbits of $\left\{\left(1, a^{2^{\alpha-1} i} b^{2^{\beta-1}}\right),\left(a^{2^{\alpha-1}}, a^{2^{\alpha-1}} b^{2^{\beta-1}}\right)\right\}$ and one orbit of $\left\{\left(1, a^{2^{\alpha-1}}\right),\left(b^{2^{\beta-1}}, a^{2^{\alpha-1}} b^{2^{\beta-1}}\right)\right\}$. By Theorem $1, P_{G}(\Omega)=3 /|\Omega|$, as required.

Remark 2 The probability that an element of metacyclic 2-groups of types (vii)-(x) fixes a set is similar to the probability that a group element fixes a set in Theorem 6 .

\section{The generalized conjugacy class graph}

This section discusses the results on the sizes of the orbits which are related to the generalized conjugacy class graph (refer to Definition 1). First, the generalized conjugacy class graph of metacyclic 2groups is found, starting with the group of type (i).

Theorem 7 Let $G$ be a group of type (i). If $G$ acts regularly on $\Omega$, then

$$
\Gamma_{G}^{\Omega_{\mathrm{c}}}= \begin{cases}K_{5}, & \beta=\gamma=1, \alpha<3, \\ K_{3}, & \text { otherwise }\end{cases}
$$

Proof: Based on Theorem 2 and when $\alpha<3$, there are five orbits all of size two. Using Definition $1, \Gamma_{G}^{\Omega_{\mathrm{c}}}$ consists of one complete component of $K_{5}$. When $\alpha>3$, there are three orbits of size two. In this case $\Gamma_{G}^{\Omega_{\mathrm{c}}}$ consists of one complete component of $K_{3}$.

Theorem 8 Let $G$ be a group of type (ii). If $G$ acts regularly on $\Omega$, then $\Gamma_{G}^{\Omega_{\mathrm{c}}}=K_{0}$.

Proof: Based on Theorem 3, there is only one orbit, namely $\Omega$. Using Definition 1 , thus $\Gamma_{G}^{\Omega_{\mathrm{c}}}$ is null.

Theorem 9 Let $G$ be a group of type (iii). If $G$ acts regularly on $\Omega$, then $\Gamma_{G}^{\Omega_{\mathrm{c}}}=K_{4}$.

Proof: According to Theorem 4, there are four orbits of size two and one orbit has size one. Using Definition 1, the generalized conjugacy class graph consists of one complete graph of $K_{4}$ and one isolated vertex, namely, $\left\{\left(a^{2^{\alpha-3} i} b, a^{2^{\alpha-3} i+2^{\alpha-1}} b\right)\right\}, 0 \leqslant i \leqslant$ $2^{\alpha}$, as claimed. 
Remark 3 Based on Remark 1, the generalized conjugacy class graph of a metacyclic 2-group of type (iv) is $K_{4}$.

The following is the generalized conjugacy class graph of a metacyclic 2-group of type (v).

Theorem 10 Let $G$ be a group of type (v). If $G$ acts regularly on $\Omega$, then

$$
P_{G}(\Omega)= \begin{cases}K_{9}, & \beta=\gamma=1 \text { and } \alpha=3 \\ K_{3}, & \text { otherwise }\end{cases}
$$

Proof: Based on Theorem 5 and when $\alpha=3$, there are nine orbits of size two. Using Definition $1, \Gamma_{G}^{\Omega_{\mathrm{c}}}$ consists of one complete graph of $K_{9}$. When $\alpha>3$, the proof is similar to the second part of the proof of Theorem 7.

Theorem 11 Let $G$ be a group of type (vi). If $G$ acts regularly on $\Omega$, then $\Gamma_{G}^{\Omega_{\mathrm{c}}}=K_{3}$.

Proof: According to Theorem 6, there are three orbits having size two. Because of the vertex adjacency of the generalized conjugacy class graph, $\Gamma_{G}^{\Omega_{c}}$ consists of one complete component of $K_{3}$.

Remark 4 Based on Remark 2, the generalized conjugacy class graph of metacyclic 2-groups of types (vii)-(x) have the same graphs, namely $K_{3}$.

\section{CONCLUSIONS}

In this paper, the probability that a group element fixes a set under regular action is found for metacyclic 2-groups of negative type of nilpotency class two and of class at least three. The results obtained are then applied to the generalized conjugacy class graph.

Acknowledgements: The authors would like to acknowledge Ministry of Education Malaysia and UTM for the Research University Fund (GUP) for vote no 08H07.

\section{REFERENCES}

1. Miller GA (1944) Relative number of non-invariant operators in a group. Proc Natl Acad Sci USA 30, 25-8.

2. Omer SMS, Sarmin NH, Erfanian A, Moradipour K (2013) The probability that an element of a group fixes a set and the group act on set by conjugation. Int J Appl Math Stat 32, 111-7.

3. Bertram EA, Herzog M, Mann A (1990) On a graph related to conjugacy classes of groups. Bull Lond Math Soc 22, 569-75.
4. Omer SMS, Sarmin NH, Erfanian A (2015) Generalized conjugacy class graph of finite non-abelian groups. AIP Conf Proc 1660, 50074.

5. Beuerle JR (2005) An elementary classification of finite metacyclic $p$-groups of class at least three. Algebra Colloq 293, 553-62.

6. Omer SMS, Sarmin NH, Erfanian A (2013) The probability that an element of a symmetric group fixes a set and its application in graph theory. World Appl Sci J 27, 48-52.

7. Omer SMS, Sarmin NH, Erfanian A (2013) The probability that an element of a group fixes a set and its graph related to conjugacy classes. J Basic Appl Sci Res 3, 369-80.

8. Bianchi M, Herzog M, Pacifici E, Saffirio G (2012) On the regularity of a graph related to conjugacy classes of groups. Eur J Combinator 33, 1402-7.

9. Moreto A, Qian G, Shi W (2005) Finite groups whose conjugacy class graphs have few vertices. Arch Math 85, 101-7.

10. You X, Qian G, Shi W (2005) A new graph related to conjugacy classes of finite groups. arXiv:math/0510015 [math.GR].

11. Moradipour K, Sarmin NH, Erfanian A (2013) On graph associated to conjugacy classes of some metacyclic 2-groups. J Basic Appl Sci Res 3, 898-902. 